\title{
CORRECTION AU VOLUME XXXV
}

\author{
M. Malric \\ Laboratoire de Probabilités et Modèles Aléatoires \\ Université Paris VI \\ 4 place Jussieu \\ 75252 PARIS Cedex 05
}

Je remercie C. Leuridan, qui m'a signalé une imprécision dans la note Filtrations quotients de la filtration brownienne (volume XXXV, pages 260-264) : si $\Gamma$ est un sous-groupe de $\mathbb{O}(d)$, la définition de la filtration quotient $\mathcal{F} / \Gamma$ varie au fil des pages, celle du bas de la page 260 n'étant jamais utilisée, et celle qui sert à établir les propositions 1, 2 et 3 n'étant pas tout à fait la même que celle qui intervient dans la démonstration de la proposition 4 .

La définition de $\mathcal{F} / \Gamma$ utilisée dans les propositions 1,2 et 3 est en fait la suivante :

Définition 1. - Une v.a. $X \operatorname{sur}(\Omega, \mathcal{A}, \mathbb{P})$ est $\mathcal{F}_{t} / \Gamma$-mesurable s'il existe une v.a. $Y$ qui soit $\mathcal{F}_{t}$-mesurable, $\Gamma$-invariante (au sens où $Y(\omega)=Y(h(\omega)$ ) pour tout $(\omega, h) \in \Omega \times \Gamma)$, et presque partout égale à $X$.

Alors que la définition qui fait marcher la démonstration de la proposition 4 est celle indiquée en haut de la page 261 :

DÉfinition 2. - Une vaa. $X$ sur $(\Omega, \mathcal{A}, \mathbb{P})$ est $\mathcal{F}_{t} / \Gamma$-mesurable si $X$ est $\mathcal{F}_{t}$ mesurable et si, pour chaque $h \in \Gamma$, on a l'égalité presque sûre $X=X \circ h$.

La seule définition raisonnable, celle qu'il faut utiliser tout au long de la note, est la définition 2. Les propositions 1, 2 et 3 restent vraies avec cette définition, tout simplement parce que les groupes considérés sont fermés dans $\mathbb{O}(d)$, donc compacts, et que, dans ce cas, les deux définitions sont équivalentes. En effet, un groupe compact admet une probabilité invariante $\mu$ (mesure de Haar), et il ne reste qu'à appliquer la technique classique de production d'objets invariants par moyennisation sous l'action du groupe : si une v.a. bornée $X$ vérifie les conditions de la définition 2 , en posant

$$
Y=\int_{\Gamma} X \circ h \mu(d h)
$$

on a $Y=Y \circ h$ pour tout $h \in \Gamma$ par invariance de la mesure de Haar; et, puisque $|Y-X|=\left|\int_{\Gamma}(X \circ h-X) \mu(d h)\right| \leqslant \int_{\Gamma}|X \circ h-X| \mu(d h)$, le théorème de Fubini donne $\mathbb{E}[|Y-X|]=0$, et la définition 1 est satisfaite également. La réciproque ( $X$ vérifie la définition $1 \Rightarrow X$ vérifie la définition 2 ) est triviale.

L'équivalence des deux définitions vaut pour tout sous-groupe de $\mathbb{O}(d)$ admettant une mesure de Haar, par exemple tout groupe $\Gamma$ borélien non négligeable pour la mesure de Haar de son adhérence $\bar{\Gamma}$. 